Original Paper

\title{
Increase of Substance P Concentration in Saliva after Pharyngeal Electrical Stimulation in Severely Dysphagic Stroke Patients - an Indicator of Decannulation Success?
}

\author{
Paul Muhle ${ }^{a}$ Sonja Suntrup-Krueger ${ }^{\mathrm{a}} \quad$ Stefan Bittner $^{\mathrm{b}} \quad$ Tobias Ruck $^{\mathrm{a}} \quad$ Inga Claus $^{\mathrm{a}}$ \\ Thomas Marian $^{\mathrm{a}} \quad$ Jens B. Schröder ${ }^{\mathrm{a}} \quad$ Jens Minnerup ${ }^{\mathrm{a}} \quad$ Tobias Warnecke $^{\mathrm{a}}$ \\ Sven G. Meuth ${ }^{\mathrm{a}}$ Rainer Dziewas ${ }^{\mathrm{a}}$ \\ aUniversity Hospital Muenster, Department of Neurology, Albert-Schweitzer-Campus 1 A, Muenster, \\ bUniversity Medical Center of the Johannes Gutenberg University Mainz, Department of Neurology, \\ Mainz, Germany
}

\section{Key Words}

Substance P - Pharyngeal electrical stimulation - PES - Stroke - Tracheostomy - Tracheal decannulation

\begin{abstract}
Background/Aims: Substance P (SP) is a neuropeptide, likely acting as a neurotransmitter in the pharyngeal mucosa enhancing the swallow and cough reflex. Pharyngeal Electrical Stimulation (PES) induces a temporary increase of salivary SP levels in healthy adults. Previous evidence suggests that post-stroke dysphagia is related to reduced SP levels. Here, we investigated the effects of PES on SP levels in severely dysphagic stroke patients and a possible link between increase of SP and treatment success. Methods: 23 tracheotomized stroke patients who could not be decannulated due to severe and persisting dysphagia according to endoscopic evaluation received PES for 10 minutes a day over three consecutive days in this prospective single-center study. If initial treatment failed, repetitive stimulation cycles were provided. Saliva samples were collected before and directly after each PES. Results: $61 \%$ of participants were decannulated after the first treatment cycle. Increase of SP levels post-stimulation was closely related to treatment success, i.e. decannulation with $79 \%$ of successfully treated patients showing increase of SP, whereas $89 \%$ of unsuccessfully treated patients had stable or decreased SP levels. Applying logistic regression analysis, increase of SP level remained the only independent predictor of decannulation after PES. All 3 repetitively treated patients showed increased SP levels when progressing from the $1^{\text {st }}$ to the $2^{\text {nd }}$ cycle, two of whom were decannulated hereafter. Conclusions: The physiological mechanism of PES may consist in restoration of sensory feedback, which is known to be crucial for the execution of a safe swallow. SP possibly acts as a biomarker for indicating response to PES.




\section{SiqínAis}

Neurosignals 2017;25:74-87

\begin{tabular}{l|l}
\hline DOI: 10.1159/000482002 & (C) 2017 The Author(s). Published by S. Karger AG, Basel \\
Published onIIne: October 17, 2017 & www.karger.com/nsg
\end{tabular}

Muhle et al.: SP Concentration Following PES in Dysphagic Stroke Patients

\section{Introduction}

Stroke-related dysphagia interferes with oral feeding and is associated with dehydration, malnutrition, prolonged hospital stay, poor long-term outcome and increased mortality [1-7]. Patients with post-stroke dysphagia have a three-times higher risk to develop pneumonia; if aspiration can be detected, the risk rises up to 11.5-times higher [4]. 1.3 $7.1 \%$ of all stroke patients are tracheotomized $[8,9]$ due to severe dysphagia with prolonged insufficient airway protection or the need for long-term ventilation [10]. The tracheostomy rate of stroke patients on the intensive care unit (ICU) is even higher and ranges between $14-35 \%$, exceeding the tracheostomy rates of mixed ICU patient collectives which lie at $10-15 \%[10-12]$.

Treatment options for stroke-related dysphagia are limited. A therapeutic potential has been described for repetitive transcranial magnetic stimulation as well as transcranial direct current stimulation, but only proof-of-principle evidence could be demonstrated so far [13-16]. Pharyngeal electrical stimulation (PES) is a neuromodulation treatment that is used to enhance cortical reorganization for the restoration of swallowing function after cerebral injury. Mapped with transcranial magnetic stimulation, Hamdy et al. showed that motor excitability and the pharyngeal cortical representation area are increased for at least 30 minutes after 10 minutes of stimulation $(10 \mathrm{~Hz})$ [17]. Subsequently the stimulation parameter with a pulse duration of $0.2 \mathrm{~ms}$ at a frequency of $5 \mathrm{~Hz}$ with $280 \mathrm{~V}$ were found to be most effective for this treatment [18]. In a magnetoencephalography (MEG) study, one session of PES was associated with changes of swallow-related brain activation within specific cortical areas such as the right primary and secondary sensorimotor cortex and the right supplementary motor area [19].

In addition to studies suggesting that PES enhances the excitability and reorganization of the human pharyngeal motor cortex, a second line of evidence focusing on the physiological mode of action of PES targets Substance P (SP). SP is a ubiquitary neuropeptide of the peripheral and central nervous system [20]. It is known to enhance the swallow and cough reflex $[21,22]$ and likely acts as a neurotransmitter in the pharyngeal mucosa in response to local stimuli. This hypothesis is further supported by a recent animal study, in which a treatment with ferulic acid for three weeks maintained laryngopharyngeal SP levels and improved swallowing reflex in hypoperfusion-induced dysphagic rats [23]. In addition, sputum levels of SP were reduced in elderly patients with aspiration pneumonia [24]. Arai et al. also proposed that aspiration after stroke may relate to low SP concentration [25]. Finally, angiotensin-converting-enzyme (ACE) inhibitors have been suggested as a possible strategy to reduce aspiration post-stroke, since these pharmaceutical agents are supposed to increase SP levels by preventing its degradation [25].

In a previous study, our group has shown that in healthy individuals, PES induces a temporary increase of SP concentration in saliva, but not in serum, whereas no effects were noticed in the sham condition [26]. In the present study, we moved this topic into the clinical context and tried to find out whether PES treatment in dysphagic stroke patients is also associated with changes in salivary SP concentrations, and, in particular, whether a potential increase in SP concentration is associated with treatment success. Since we previously had shown that PES enhances remission of dysphagia in severely affected tracheotomized stroke patients, which subsequently allowed post-treatment decannulation [27] in the majority of cases, we chose a comparable cohort as a target group here.

\section{Material and Methods}

Study design and setting

A prospective single-center study was conducted recruiting patients who were admitted to the neurological ICU of the University Hospital Muenster due to an ischemic or hemorrhagic stroke between October 2014 and August 2015. Participants had to be completely weaned from the ventilator and had to be able to 
stay alert for at least 15 minutes. Patients were eligible to be included in this study if they were tracheotomized and suffered from severe and persisting dysphagia that would not allow for decannulation (decannulation-criteria see "dysphagia assessment"). Exclusion criteria were the presence of implanted electronic devices. Screening, recruitment and FEES were performed either on the ICU or on our stroke unit. Every eligible patient received at least one FEES according to the protocol proposed by Warnecke and co-workers [28]. Opposed to other trials in this area [27, 29, 30], no further exclusion criteria were adopted. In particular, patients with pre-existing dysphagia or conditions known to be related to dysphagia (neurodegenerative diseases, neuromuscular disorders, malignancies of the oral cavity or throat, history of stroke) were all allowed to participate in this trial. As increasing age could be identified as a risk factor for acute post-stroke dysphagia [31] and increasing age goes along with a higher risk to suffer from comorbidities, further knowledge on the role of these comorbidities on the efficiency of PES is needed, especially with regard to a more general clinical application of PES in the future. Patients who could not be decannulated after the first treatment cycle were offered additional treatment cycles (Fig. 1). Being in a neurointensive care setting, all participants were continuously monitored with ECG, blood-pressure reading and pulse oximetry - also during the PES treatment. Data on age, sex, National Institutes of Health Stroke Scale (NIH-

SS) on admission, type and site of stroke, stroke etiology classified according to the TOAST (Trial of Org 10172 in Acute Stroke Treatment) criteria [32], vascular risk factors and premorbid conditions being related to dysphagia were obtained. We kept records regarding intubation, acute stroke treatment (i.e. thrombolytic therapy, mechanical recanalization, neurosurgical intervention), time from orotracheal intubation to tracheotomy, total time of mechanical ventilation, and time from stroke onset as well as termination of ventilation until study inclusion. Informed consent was obtained from all patients or, in case the patient's communication was impaired, from their next of kin. The study was approved by the local ethics committee at the University of Muenster.

\section{Dysphagia assessment}

Fiberoptic endoscopic evaluation of swallowing (FEES) was performed by a trained neurologisttogetherwith a speechlanguage therapist according to our protocol for standardized endoscopic swallowing evaluation for tracheotomy decannulation in critically ill neurologic patients [28]. In this protocol, the management of secretions, spontaneous swallow frequency,
Enrollment

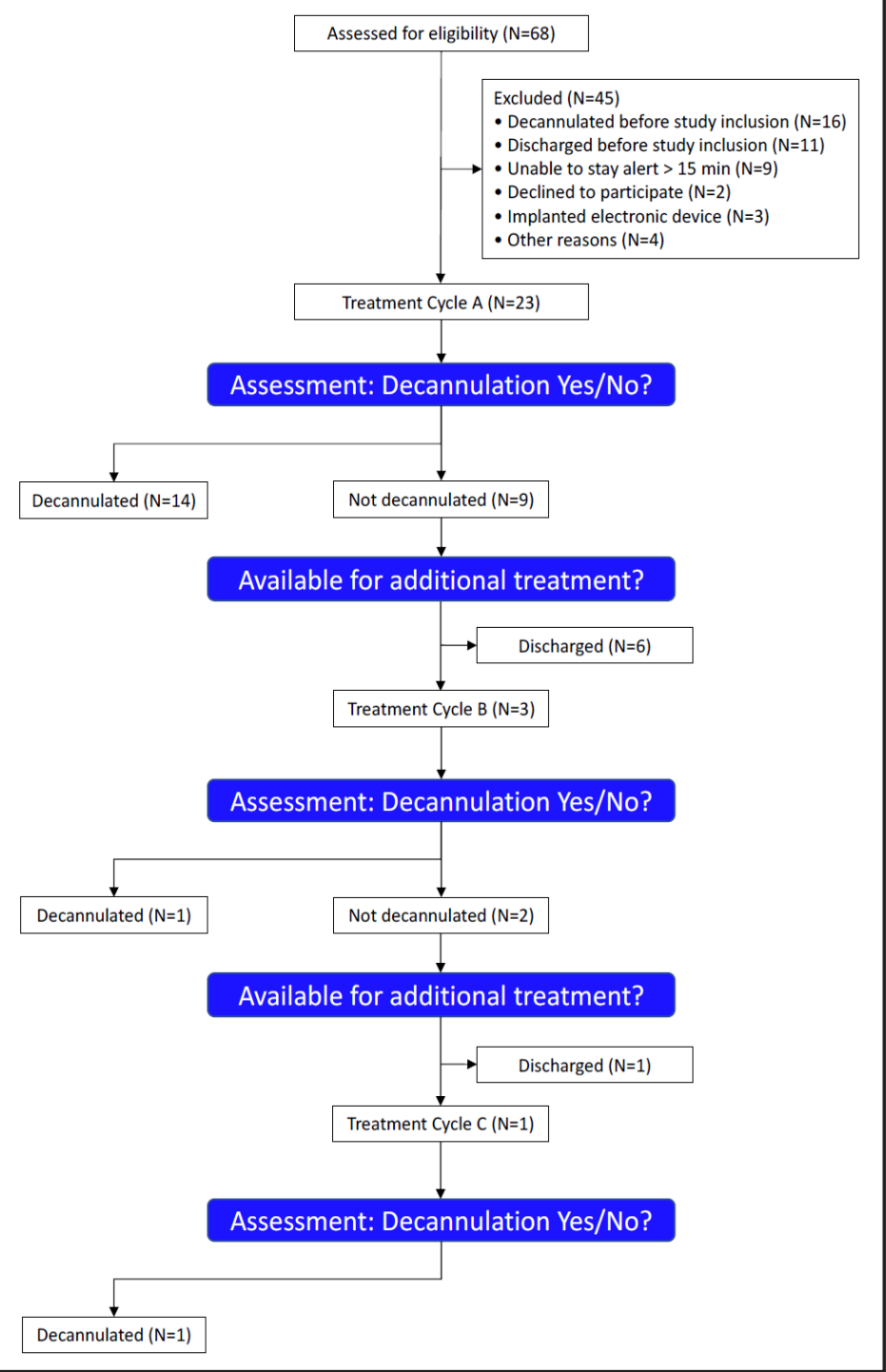

Fig. 1. Study protocol. 
laryngeal sensitivity and cough are evaluated in a stepwise manner. If a patient failed one of the single steps according to the decisional flowchart [28], decannulation was regarded as unsafe. To perform FEES, we used a 3.1-mm-diameter flexible fiberoptic rhinolaryngoscope (11101 RP2, Karl Storz, Tuttlingen, Germany), a combined light source and camera system delivering videos in standard definition quality (rpCAM-X, Rehder/Partner Medizintechnik, Hamburg, Germany) and a Medical Panel PC (WMP-226, Wincomm Corporation, Taiwan), which allows to display and record the examination. Additionally, the video material was stored on an external hard disc in avi format. The entire system, including the endoscope, light source/camera delivering system and Panel PC, is contained on a portable instrument cart.

\section{Pharyngeal electrical stimulation (PES)}

The Phagenyx ${ }^{\mathrm{TM}}$ catheter system and base station (Phagenesis, Ltd, UK) were used to deliver the stimulation. The system contains a nasogastric feeding tube that houses a pair of bipolar titanium ring electrodes that are used for the treatment. The feeding tube was inserted transnasally and the electrodes, which are $10 \mathrm{~mm}$ apart, were positioned in the middle pharynx under FEES control. To perform the treatment, the catheter was connected to the base station and stimuli of $0.2 \mathrm{~ms}$ pulse duration at a frequency of $5 \mathrm{~Hz}$ with $280 \mathrm{~V}$ were delivered. These parameters were previously described to be most effective for this treatment [18]. The current intensity ( $\mathrm{mA}$ ) was adjusted individually in every session. Therefore, prior to the actual intervention, the perceptual threshold (PT) and the maximum tolerated threshold (MTT) were determined repeatedly by slowly increasing the current. The average values of three trials were taken into account for the calculation of the optimal stimulation intensity according to the formula PT $+0.75 \times$ (MTT - PT) [18] and documented for each treatment session. The stimulation was delivered for a total of 10 minutes and repeated daily for three consecutive days. In case dysphagia did not improve after the first treatment cycle (treatment cycle A), subsequent treatments could be delivered (treatment cycle B, C etc.). The stimulation catheter remained in place over this period of time and was used as a regular feeding tube between treatment sessions. PES treatment success was evaluated the day after each treatment cycle was completed. If a patient failed decannulation criteria according to the FEES-based protocol and was available for another treatment cycle, PES treatment started within $48-72 \mathrm{~h}$ after FEES. None of the patients received other dysphagia-related treatment during participation in the study.

\section{Substance $P$ analysis}

Saliva samples were collected at the bedside on the ICU using a salivette (Sarstedt, Nuembrecht, Germany) twice per stimulation session, first right before determining the PT and secondly immediately after the treatment had been applied. In order to collect the saliva, two salivettes were placed into the cheek pouches for $1-2$ minutes. Directly after collecting the saliva, the salivettes were centrifuged at $4110 \mathrm{~g}$ for 5 minutes. Supernatants were stored in a deep freezer at $-20^{\circ} \mathrm{C}$ until further analysis. We used a commercially available ELISA-type immunoassay (SP Immunoassay, catalogue no. KGE007; R\&D Systems, Minneapolis, MN, USA) to obtain salivary SP concentration.

\section{Statistical analysis}

All analyses were performed using IBM SPSS Statistics 24 (IBM Corporation, Armonk, New York, USA). Patients showing improvement of dysphagia after PES were compared to those remaining tracheotomized because of no or insufficient remission of swallowing dysfunction. Because of the high variability of individual SP concentration data, these were normalized using the respective pre-stimulation value as reference. Descriptive statistics were used to quantify patient characteristics. The data are presented as frequencies for categorical variables and mean \pm standard deviation for continuous variables. Categorical variables were tested using the Fisher exact test. Presence of a normal distribution of continuous variables was confirmed by Kolmogorov-Smirnov statistics. Normally distributed continuous variables were compared with the Student's t-test. The Mann-Whitney $U$ test was used as an analogue non-parametric test. Logistic regression analysis was applied to identify independent predictors for decannulation. In the logistic regression analysis results of SP increase were dichotomized with " 1 " being overall average increase and " 0 " being overall decrease/stable concentration of SP during treatment cycle A.

\section{KARGER}




\section{Results}

\section{Patients' characteristics}

Patients' characteristics are summarized in Table 1. A number of comorbidities known to likely cause dysphagia were found in the patient collective: One patient suffered from Parkinson's disease and one patient was diagnosed with dementia. Five patients had a history of stroke, one of whom was additionally diagnosed with dementia; one of whom additionally suffered from polymyalgia rheumatica. One patient had a history of oropharyngeal cancer and was treated 10 years before PES treatment.

\section{Pharyngeal electrical stimulation}

During catheter insertion or PES, no adverse events occurred. Calculated and delivered optimum stimulation intensity during stimulation cycle A was $28.28 \mathrm{~mA}$. The successfully treated group of patients had a lower average stimulation intensity in comparison to patients without treatment success $(25.71 \pm 9.05 \mathrm{~mA}$ vs. $32.26 \pm 10.27 \mathrm{~mA})$; however, the respective differences were not statistically significant $(\mathrm{p}=0.123 ; \mathrm{t}=1.607)$. Also, no

Table 1. Epidemiological and clinical parameters of the study population (SD = standard deviation; $\mathrm{h}$ = hours; $\mathrm{d}$ = days; LOS = length of stay; ICU = intensive care unit; ACE = angiotensin-converting-enzyme; FEES = fiberoptic endoscopic evaluation of swallowing; PES = pharyngeal electrical stimulation)

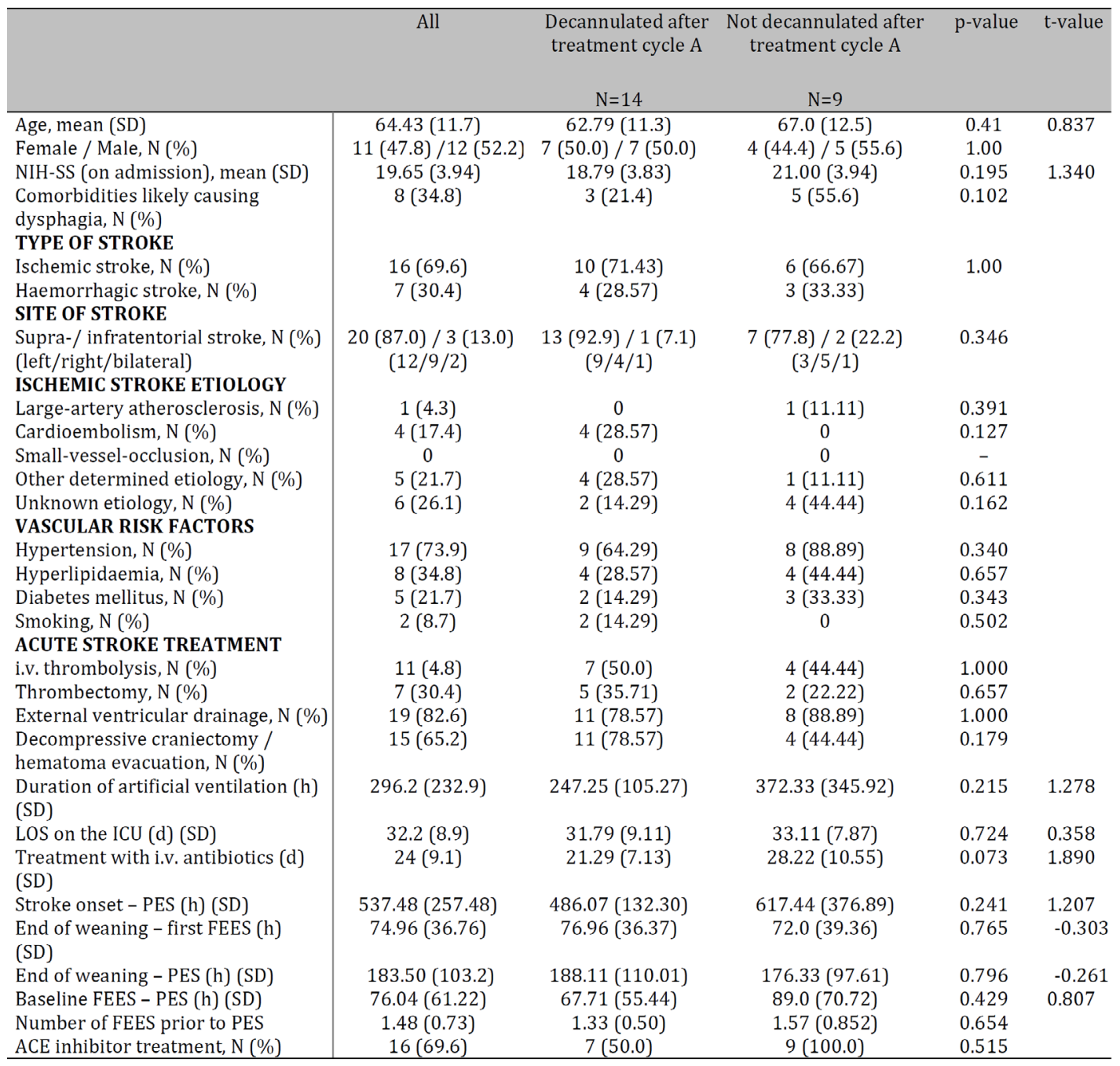




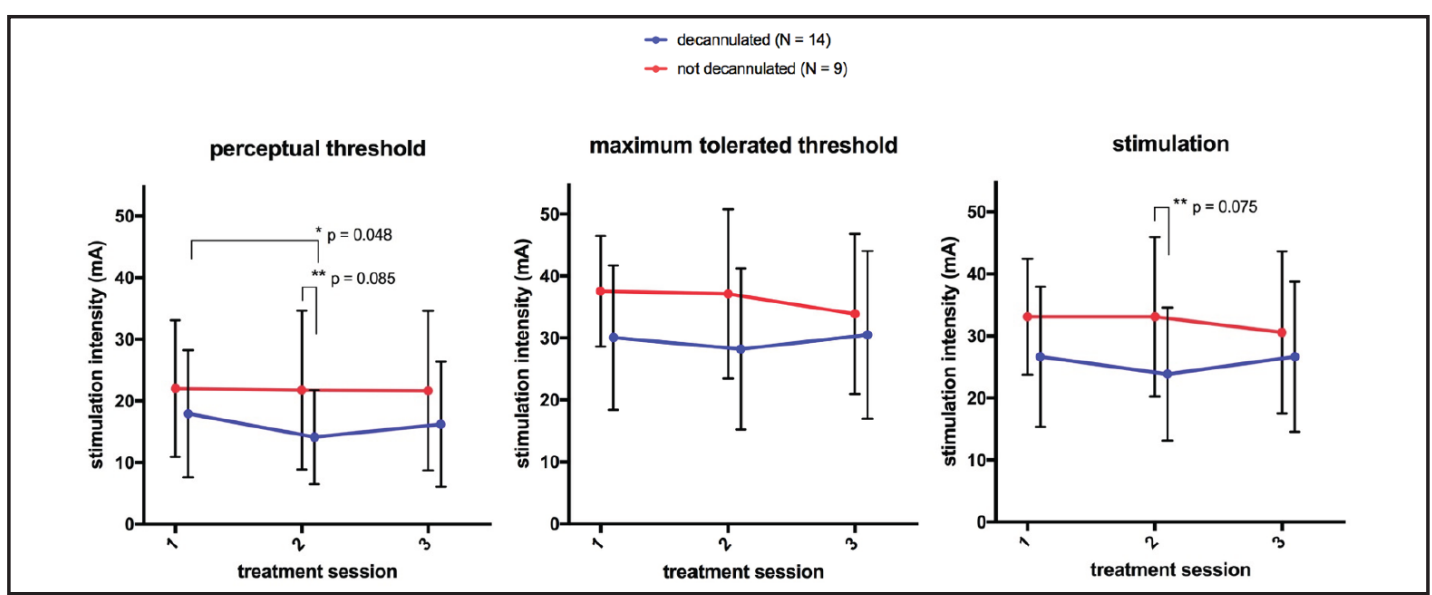

Fig. 2. Perceptual thresholds, maximum tolerated thresholds and stimulation intensities for each treatment session during treatment cycle A separated by groups (decannulated and not decannulated) $(\mathrm{mA}=\mathrm{milliam}$ pere).

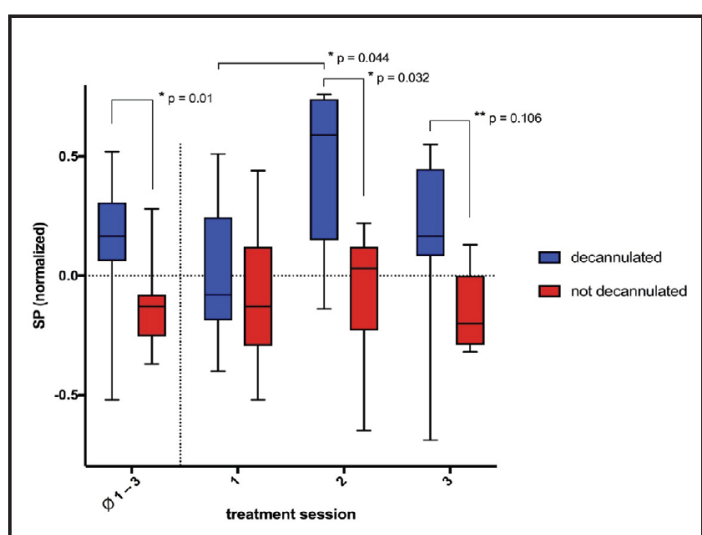

Fig. 3. Mean increase of substance P level in saliva during treatment cycle A separated by groups (decannulated/not decannulated) (median, 95-confidence interval and standard deviation); treatment session 1: decannulated $\mathrm{N}=12$; not decannulated $\mathrm{N}=8$; treatment session 2: decannulated $\mathrm{N}=8$; not decannulated $\mathrm{N}=7$; treatment session 3 : decannulated $\mathrm{N}=12$; not decannulated $\mathrm{N}=7$.

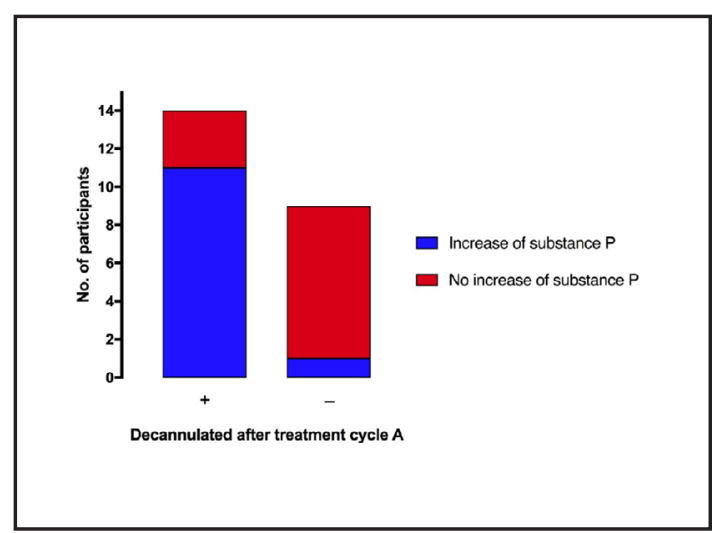

Fig. 4. Distribution of patients with an increase or stable/decreasing levels of substance P during treatment cycle A separated by groups (decannulated/not decannulated).

significant differences were found for PT and MTT between the groups. Successfully treated patients showed a significant decrease of PT intensity between treatment A1 and A2 $(\mathrm{p}=0.048 ; \mathrm{t}=2.181)$. The average current intensities per group on PT, MTT and the stimulation are detailed in Fig. 2.

\section{Treatment results}

Within $72 \mathrm{~h}$ after finishing PES-treatment cycle A, 14 of 23 participants (60.9\%) could be decannulated according to the previously mentioned protocol [28]. Three patients who showed no treatment success received further PES (s. "Multiple stimulation cycles"). Comparing patients with and without treatment success after treatment cycle A, no significant differences were found with regard to demographic and clinical characteristics (s. Table 1). Excluding patients with comorbidities that likely cause dysphagia, 11 of 15 (73.3\%) patients could be decannulated after treatment cycle A. During hospitalization at our clinic (34.83 \pm 10.65 days in mean), none of the participants had to be re-cannulated or intubated. 
Table 2. Logistic regression analysis of decannulation success

\begin{tabular}{|c|c|c|c|c|c|c|c|c|}
\hline & \multirow[t]{2}{*}{$\begin{array}{l}\text { Regression } \\
\text { coefficient B }\end{array}$} & \multirow[t]{2}{*}{$\begin{array}{c}\text { Standard } \\
\text { error }\end{array}$} & \multirow[t]{2}{*}{ Wald } & \multirow[t]{2}{*}{ df } & \multirow[t]{2}{*}{ Significance } & \multirow[t]{2}{*}{$\operatorname{Exp}(B)$} & \multicolumn{2}{|c|}{$95 \%$ confidence interval } \\
\hline & & & & & & & lower bound & upper bound \\
\hline Age & -0.023 & 0.112 & 0.042 & 1 & 0.837 & 0.977 & 0.785 & 1.216 \\
\hline Gender & -1.030 & 2.862 & 0.129 & 1 & 0.719 & 0.357 & 0.001 & 97.596 \\
\hline Comorbidity likely causing dysphagia & -3.829 & 3.677 & 1.085 & 1 & 0.298 & 0.022 & 0.000 & 29.291 \\
\hline NIH-SS & -0.406 & 0.259 & 2.448 & 1 & 0.118 & 0.667 & 0.401 & 1.108 \\
\hline Duration of artificial ventilation & -0.018 & 0.018 & 1.049 & 1 & 0.306 & 0.982 & 0.948 & 1.017 \\
\hline Increase of substance $\mathrm{P}$ & 4.154 & 2.090 & 3.950 & 1 & 0.047 & 63.705 & 1.059 & 3831.329 \\
\hline Constant & 15.762 & 12.173 & 1.677 & 1 & 0.195 & 7003891.11 & & \\
\hline
\end{tabular}

Fig. 5. Relative increase of substance $P$ in multiply stimulated patients for each treatment cycle (mean and standard deviation).

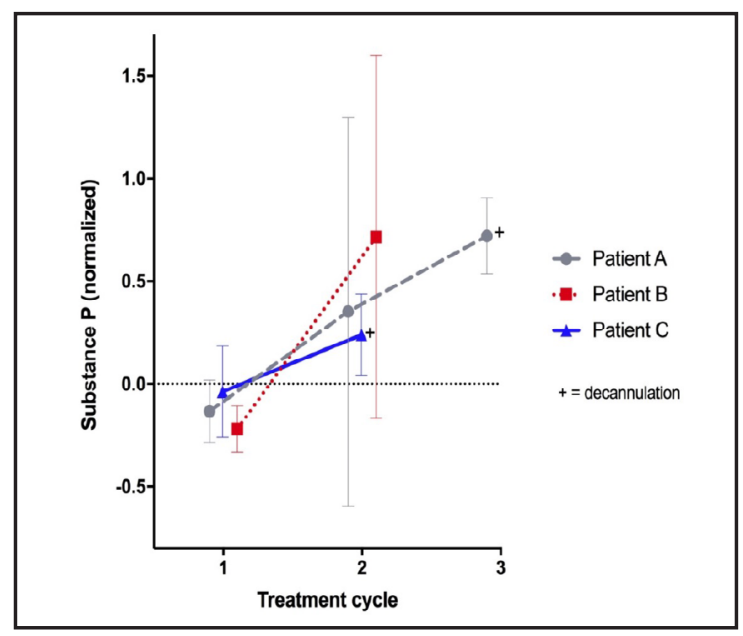

\section{Substance P}

Saliva samples were collected in each patient right before and after each PES. Sufficient sample material for further analysis could be collected in 20 patients during the first treatment (A1), and in 15 and 18 patients during the second (A2) and third treatment sessions (A3) respectively. As shown in Fig. 3, successful PES was associated with an increase of SP-values post- over pre-stimulation, whereas in non-successfully treated patients, there was a small decline in SP-values. In this first group, SP increase was particularly pronounced after the second stimulation $(+28 \%)$ and to a lesser extent after the third stimulation $(+11 \%)$ of treatment cycle A, whereas no SP increase was seen after the first stimulation. During the three stimulation sessions, no increase of mean SP level was observed in patients with treatment failure. With regard to the entire study population the overall increase of SP during treatment cycle A (normalized data) was $0.09 \pm 0.58$, respectively $0.32 \pm 0.59$ in the group of patients showing treatment success and $-0.28 \pm 0.35$ in the group of patients who could not be decannulated $(\mathrm{p}=0.01 ; \mathrm{t}=-2.74)$. Although the magnitude of SP increases differed between patients with PES treatment success, 11 of 14 patients $(78.6 \%)$ showed an increase of mean SP from all three stimulations. Conversely, in 8 out of 9 patients $(88.9 \%)$ with treatment failure, mean SP levels remained stable or decreased (Fig. 4) $(p=0.01)$.

\section{Predictors of treatment success}

As inferred from logistic regression analysis (Table 2) including age, gender, preexisting dysphagia/comorbidities that likely cause dysphagia, NIH-SS, duration of artificial ventilation and increase of SP, increase of SP remained the only significant predictor of an improved swallowing function $(\mathrm{p}=0.047)$. 


\section{Multiple stimulation cycles}

Two of three patients with multiple stimulation cycles were finally decannulated; two received one additional, the third patient received two additional treatment cycles. The patient without treatment success was treated with one additional treatment cycle and was not available for further treatment due to hospital discharge. The course of SP concentration for each treatment cycle is depicted in Fig. 5. All three patients showed an increase of SP when progressing from one to the next treatment cycle.

\section{Angiotensin-converting-enzyme (ACE) inhibitors}

SP concentration in saliva did not differ significantly between patients with or without ACE inhibitor treatment (16 vs. 7 patients) prior or during PES ( $p=0.662$ ). There was no significant difference between the groups with regard to PES-treatment success during treatment cycle A $(\mathrm{p}=0.657)$.

\section{Discussion}

In this proof-of-principle study on tracheotomized stroke patients, we studied the influence of PES on SP saliva concentrations and its relation to treatment-related decannulation success. The main finding of this study was that an increase of SP directly after stimulation was closely related to improved swallowing function. This finding was corroborated by logistic regression analysis; here an increased level of SP was the only independent predictor of successful decannulation, whereas no correlation was observed for age and sex, NIH-SS on admission, dysphagia-wise relevant comorbidities and lengths of artificial ventilation. As the second main finding, the significance of PES-associated increase of SP was also observed in a small cohort of three patients that received multiple stimulation cycles. All three patients showed an increase of mean SP levels when progressing from the first to the second cycle. In one patient, this trend was also valid when a third cycle of PES was applied. Two of these three patients could be decannulated after repetitive PES.

As detailed above, SP has previously been shown to be intimately involved in the physiology and pathophysiology of swallowing. The present study expands on these findings by suggesting that determination of salivary SP levels during a therapeutic intervention might indeed work as biomarker indicating treatment efficacy. Thus, $78.6 \%$ of patients who were treated successfully by PES showed a post-stimulation increase of SP, while on the other hand, an even slightly higher proportion of patients (88.9\%) without clinical improvement of dysphagia had stable or decreased SP levels. These results are also in line with a very recent and methodologically different study [33] that evaluated the effect of capsaicin on different subjective and objective measures of the oropharyngeal and esophageal swallow in a cohort of elderly patients complaining of dysphagic symptoms. The study was conducted as sham-controlled trial and saliva SP levels were determined before and after medication was given. Similar to the results presented here, treatment with capsaicin was followed by both an improvement of swallowing physiology and an increase of salivary SP levels.

The findings of our study are also instrumental to expand our understanding of the mode of action of PES. The proof-of-principle studies by Hamdy and co-workers demonstrated that PES enhances the excitability and reorganization of the pharyngeal motor cortex, thereby promoting the notion of stimulation-associated effects on the level of the central nervous system [17, 34]. The results of this and of a preceding study by our group suggest that in addition to stimulation driven motor-cortical reorganization, restoration of peripheral sensory feedback may play a role here. Thus, in line with our previous study [27], we observed a slight decline of stimulation intensities in patients showing clinically relevant treatment response, which might be interpreted as partial recuperation of the afferent sensory system induced by PES. More importantly, however, the close relation of SP increase and successful PES treatment alludes to a causal role of SP in the underlying physiological mechanism. SP 
occurs ubiquitary in the peripheral nervous system, is found in particular in C and A $\delta$ fiber terminals in the pharyngeal mucosa and colocalizes there with TRPV1 channels which have been suggested to play an important role in the perception of noxious stimuli and prevention of aspiration $[35,36]$. Thus, it is conceivable that PES triggered release of SP produces peripheral sensitization of sensory neurons, which then facilitates the motor swallow response in the upstream swallowing network. Interestingly, this reasoning suggests a dual effect of PES on dysphagia in this specific patient cohort. First and most obvious, the patients included in this trial suffered from "classical" post-stroke dysphagia due to the massive brain damage causing direct disruption of the cortical swallowing network. Therefore, PES might have been instrumental in improving deglutition by its above-mentioned ability to enhance cortical reorganization of the pharyngeal motor cortex. Second, and less apparent at first sight, dysphagia in tracheotomized stroke patients is - at least in part - also due to non-stroke specific conditions that are generally associated with intensive care treatment. Both lesions to the pharyngeal and laryngeal mucosa caused by endotracheal, tracheal and nasogastric tubes and critical illness neuropathy as a possible consequence of long-lasting ICU treatment are prone to cause an impairment of pharyngeal and laryngeal sensory afferents. With regard to this issue, PES might have helped recovering swallowing function along the abovementioned pathway of peripheral SP release and subsequent recruitment of afferent sensory pathways of the swallowing network.

Obviously, based on the data presented here, it is impossible to confidently conclude which of the two putative physiological mechanisms of PES have a greater impact on recovery of swallowing function in the collective of tracheotomized stroke patients. However, apart from the close correlation of SP increase and treatment success, the short time interval between PES and observed improvement of swallowing function suggests that the second mechanism targeting sensory feedback should not be underestimated. Thus, motor-cortical reorganization subsequent to PES might indeed show its full effect only after several days post-treatment. It was basically this assumption that made the authors of all previous PES studies recruiting "regular" stroke patients to determine the primary endpoint around 14 days after the start of PES $[30,37,38]$. Opposed to this, the restoration of afferent sensory feedback happens probably much faster. Apart from the observations made in the present trial, it has also been shown that PES is associated with immediate effects on the swallowing network [17-19].

Based on the proof-of-principle study in 2010 [37], PES is usually applied as single treatment cycle comprising 3 stimulations on 3 consecutive days [27, 30, 38]. The present study provides the first data to re-evaluate this standard procedure. Thus, because of insufficient treatment success, three patients in this trial were exposed to multiple stimulation cycles. Interestingly, all of them showed increasing SP concentrations when moving from one treatment cycle to the next, ultimately resulting in successful decannulation in two of them. Although this finding is clearly very preliminary, it nevertheless suggests that patients with severely impaired sensory feedback as the main feature of disordered swallowing should be particularly considered for re-treatment with PES. Remarkably, this idea has also been adopted in the multicenter PHAST TRAC trial (Benefit of PHAryngeal electrical STimulation for early decannulation in TRACheotomised stroke patients with neurogenic dysphagia: a prospective randomized single-blinded interventional study) that randomizes tracheotomized stroke patients in a first step to PES or sham treatment [29]. In case that patients are not ready for decannulation after this initial study period, they will receive an unblended cycle of PES, which means that at least part of the study cohort will be exposed to two cycles of stimulation. Apart from that, the also ongoing PHADER registry (PHAryngeal electrical stimulation for treatment of neurogenic Dysphagia: a European Registry [39]; ISRCTN87110165[40]) also allows for repetitive stimulation cycles.

With regards to clinically relevant endpoints, PES has been evaluated in several clinical trials that provide heterogeneous results so far. In non-intubated stroke patients, two smaller randomized controlled trials and one related meta-analysis showed significant 
improvement of swallowing function and in particular a reduction of aspiration risk post PES [37, 38, 41]. PES was also used with good success in a small trial including dysphagic patients suffering from multiple sclerosis [42]. The comparatively larger STEPS trial (Swallowing Treatment Using Electrical Pharyngeal Stimulation) recruited 162 patients with post-stroke dysphagia from 20 sites [30]. PES treatment was applied in median 13.4 days post-stroke (standard deviation: 9.7 days). As opposed to the previous studies targeting the same patient population, STEPS showed no benefit of PES over sham stimulation with regards to improvement of swallowing safety. Finally, as mentioned above, PES was evaluated in a single-center randomized controlled trial in tracheotomized stroke patients [27]. In that trial, $75 \%$ of patients in the stimulation group were decannulated after the treatment as opposed to $20 \%$ of the control group. In addition, $71 \%$ of the control patients regained a sufficient swallowing function so as to enable decannulation after an unblinded stimulation during the follow-up period.

In comparison to the randomized trial on tracheotomized stroke patients, the decannulation rate was noticeably lower in the present non-randomized study (61\% vs. $75 \%)$. The reason for this might be seen in differences of the two patient cohorts. Thus, keeping in mind that overall stroke severity is among the most important risk factors for post-stroke dysphagia and its complications [43-45], the present study featured again a severely handicapped patient cohort with a higher rate of intracerebral hemorrhage $(30 \%$ vs. 10\%). Most importantly, the present trial allowed for a nearly unrestricted inclusion of patients. Thus, patients with pre-existing dysphagia and/or patients having a history of diseases typically increasing the risk of dysphagia, both of whom are usually excluded from this kind of trial, were eligible for study participation. Therefore, the cohort of the present study featured 8 patients with a dysphagia-wise critical premorbid condition and, unsurprisingly, these patients indeed showed a worse response to PES treatment.

Finally, the role of ACE inhibitors in the context of swallowing safety needs to be briefly addressed, since ACE inhibitors were a frequent co-medication of patients also in the present trial. In principle, ACE inhibitors are known to prevent degradation of SP and have therefore been associated with increased levels of that neuropeptide [46]. Consequently, referring to the ability of SP to enhance protective reflexes, ACE inhibitor treatment has been discussed as a possible strategy to reduce aspiration after stroke [47]. Interestingly, in line with this reasoning, two Asian observational studies found reduced pneumonia rates in hypertensive stroke patients treated with ACE inhibitors in comparison to patients managed with other antihypertensive drugs $[48,49]$, a conclusion that was also uphold in a meta-analysis [50, 51]. However, in more recent trials, this assumption was decisively questioned with two case-control studies and one randomized-controlled trial failing to show any evidence of a putative protective role of ACE inhibitors in dysphagic stroke and non-stroke patients [5254]. These latter findings are in line with our own results, where concomitant treatment with ACE inhibitors did not have an effect on SP levels or stimulation success.

Several limitations of the present study need to be addressed. First, the number of patients included in this trial, although comparable to other studies in this area, is still too small to draw any firm conclusions. Second, due to insufficient saliva production, it was not possible to get saliva samples prior and post each stimulation in every patient, which might have introduced a bias into the data analysis. Third, each patient of this cohort received a considerable number of different drugs. Whereas a possible relation of SP levels and ACE inhibitors was specifically analyzed and no correlation was found, it has to be acknowledged that other pharmaceutical agents might have biased study results. Fourth, in some patients a decline of SP levels after stimulation was found. The physiological implication of this finding is unclear at the moment and needs to be elucidated in future trials. Finally, we aimed at recruiting a less selected study collective than in previous trials to achieve more generalizable results. We need to concede however that the advantage of this approach might at least in part have been offset by a possible dilution of the PES-SP interaction. 


\section{Conclusion}

In conclusion, this prospective observational trial suggests that PES is instrumental in treating dysphagia in tracheotomized stroke patients. The underlying physiological mechanism of PES may consist in a restoration of sensory afferents critically needed for the execution of a safe and efficient swallow. SP may work as biomarker indicating the patient's potential responsiveness to PES.

\section{Sources of Funding}

The work was supported by the Deutsche Forschungsgemeinschaft [grant number SU 922/1-1, W01425/6-1, DZ 78/1-1].

\section{Abbreviations}

ACE (angiotensin-converting-enzyme); FEES (fiberoptic endoscopic evaluation of swallowing); ICU (Intensive Care Unit); MEG (magnetoencephalography); MTT (maximum tolerated threshold); NIH-SS (National Institutes of Health Stroke Scale); PES (pharyngeal electrical stimulation); PT (perceptual threshold); SP (Substance P).

\section{Acknowledgements}

We would like to thank our speech-language therapists Sigrid Colbow, Lisa-Marie Hufelschulte, Stefan Oelenberg, Sonja Sauer and Christina Wüller who helped us performing FEES.

\section{Disclosure Statement}

P. Muhle has received lecture fees and travel compensation from Olympus and a travel grant from the European Academy of Neurology (EAN). S. Suntrup-Krueger has received a grant from Deutsche Forschungsgemeinschaft (DFG), a travel grant from the European Federation of Neurological Societies (EFNS) and lecture fees from Abbvie, Fresenius Kabi, Nestle and Boehringer Ingelheim. S. Bittner has received grants from Deutsche Forschungsgemeinschaft (DFG) and Hertie-Stiftung and lecture fees from Biogen Idec, Genzyme, Roche, Merck Serono and TEVA. T. Ruck received travel expenses and financial research support from Genzyme and Novartis and received honoraria for lecturing from Roche, Merck, Genzyme, Biogen, and Teva. J.B. Schroeder received speaker's honoraria from Abbvie Inc. J. Minnerup has received grants from Deutsche Forschungsgemeinschaft (DFG), Bundesministerium für Bildung und Forschung (BMBF), Else Kröner-Fresenius-Stiftung, EVER Pharma Jena GmbH, Ferrer International, travel grants from Boehringer Ingelheim and speaking fees from Bayer Vital. T. Warnecke has received lecture fees from Abbvie, Teva, Bayer, and UCB, worked as a consultant for Abbvie and UCB, and received a research grant from Deutsche Parkinson Vereinigung e.V. (dPV). S.G. Meuth has received honoraria for lecturing and travel expenses for attending meetings and has received financial research support from Bayer, Bayer Schering, Biogen Idec, Genzyme, Merck Serono, MSD, Novartis, Novo Nordisk, Sanofi-Aventis, Teva, and UCB. R. Dziewas received lecture fees from Nutricia, Fresenius Kabi, Boehringer Ingelheim, Bayer, Pfizer, Daiichi Sankyo, worked as a consultant for Nestle, Nutricia, Daichii Sankyo, Pfizer, Boehringer Ingelheim and InfectoPharm, and is member of the clinical advisory board of Phagenesis. He received research grants from Deutsche Forschungsgemeinschaft. I. Claus. and T. Marian declare no conflicts of interest. 


\section{SIGINAIS}

Neurosignals 2017;25:74-87

\begin{tabular}{l|l}
\hline DOI: 10.1159/000482002 & $\begin{array}{l}\text { C } 2017 \text { The Author(s). Published by S. Karger AG, Basel } \\
\text { www.karger.com/nsg }\end{array}$ \\
\hline
\end{tabular}

Muhle et al.: SP Concentration Following PES in Dysphagic Stroke Patients

\section{References}

1 Hannawi Y, Hannawi B, Rao CP, Suarez JI, Bershad EM: Stroke-associated pneumonia: major advances and obstacles. Cerebrovasc Dis 2013;35:430-443.

-2 Hoffmann S, Malzahn U, Harms H, Koennecke HC, Berger K, Kalic M, Walter G, Meisel A, Heuschmann PU, Berlin Stroke R, the Stroke Register of Northwest G: Development of a clinical score (A2DS2) to predict pneumonia in acute ischemic stroke. Stroke 2012;43:2617-2623.

3 Smithard DG, Smeeton NC, Wolfe CD: Long-term outcome after stroke: does dysphagia matter? Age Ageing 2007;36:90-94.

4 Martino R, Foley N, Bhogal S, Diamant N, Speechley M, Teasell R: Dysphagia after stroke: incidence, diagnosis, and pulmonary complications. Stroke 2005;36:2756-2763.

$>5$ Kumar S, Selim MH, Caplan LR: Medical complications after stroke. Lancet Neurol 2010;9:105-118. Foley NC, Martin RE, Salter KL, Teasell RW: A review of the relationship between dysphagia and malnutrition following stroke. J Rehabil Med 2009;41:707-713.

7 Gonzalez-Fernandez M, Ottenstein L, Atanelov L, Christian AB: Dysphagia after Stroke: an Overview. Curr Phys Med Rehabil Rep 2013;1:187-196.

8 Roth EJ, Lovell L, Harvey RL, Bode RK, Heinemann AW: Stroke rehabilitation: indwelling urinary catheters, enteral feeding tubes, and tracheostomies are associated with resource use and functional outcomes. Stroke 2002;33:1845-1850.

-9 Walcott BP, Kamel H, Castro B, Kimberly WT, Sheth KN: Tracheostomy after severe ischemic stroke: a population-based study. J Stroke Cerebrovasc Dis 2014;23:1024-1029.

10 Bosel J: Tracheostomy in stroke patients. Curr Treat Options Neurol 2014;16:274.

11 Kurtz P, Fitts V, Sumer Z, Jalon H, Cooke J, Kvetan V, Mayer SA: How does care differ for neurological patients admitted to a neurocritical care unit versus a general ICU? Neurocrit Care 2011;15:477-480.

12 Pelosi P, Ferguson ND, Frutos-Vivar F, Anzueto A, Putensen C, Raymondos K, Apezteguia C, Desmery P, Hurtado J, Abroug F, Elizalde J, Tomicic V, Cakar N, Gonzalez M, Arabi Y, Moreno R, Esteban A, Ventila Study G: Management and outcome of mechanically ventilated neurologic patients. Crit Care Med 2011;39:14821492.

13 Shigematsu T, Fujishima I, Ohno K: Transcranial direct current stimulation improves swallowing function in stroke patients. Neurorehabil Neural Repair 2013;27:363-369.

14 Kumar S, Wagner CW, Frayne C, Zhu L, Selim M, Feng W, Schlaug G: Noninvasive brain stimulation may improve stroke-related dysphagia: a pilot study. Stroke 2011;42:1035-1040.

15 Park JW, Oh JC, Lee JW, Yeo JS, Ryu KH: The effect of 5Hz high-frequency rTMS over contralesional pharyngeal motor cortex in post-stroke oropharyngeal dysphagia: a randomized controlled study. Neurogastroenterol Motil 2013;25:324-e250.

16 Khedr EM, Abo-Elfetoh N, Rothwell JC: Treatment of post-stroke dysphagia with repetitive transcranial magnetic stimulation. Acta Neurol Scand 2009;119:155-161.

17 Hamdy S, Rothwell JC, Aziz Q Singh KD, Thompson DG: Long-term reorganization of human motor cortex driven by short-term sensory stimulation. Nat Neurosci 1998;1:64-68.

18 Fraser C, Power M, Hamdy S, Rothwell J, Hobday D, Hollander I, Tyrell P, Hobson A, Williams S, Thompson D: Driving plasticity in human adult motor cortex is associated with improved motor function after brain injury. Neuron 2002;34:831-840.

19 Suntrup S, Teismann I, Wollbrink A, Winkels M, Warnecke T, Pantev C, Dziewas R: Pharyngeal electrical stimulation can modulate swallowing in cortical processing and behavior - magnetoencephalographic evidence. Neuroimage 2015;104:117-124.

20 Niel JP: [Role of substance P in the nervous system control of digestive motility]. Arch Int Physiol Biochim Biophys 1991;99:A65-76.

21 Imoto Y, Kojima A, Osawa Y, Sunaga H, Fujieda S: Cough reflex induced by capsaicin inhalation in patients with dysphagia. Acta Otolaryngol 2011;131:96-100.

22 Jin Y, Sekizawa K, Fukushima T, Morikawa M, Nakazawa H, Sasaki H: Capsaicin desensitization inhibits swallowing reflex in guinea pigs. Am J Respir Crit Care Med 1994;149:261-263. 
23 Asano T, Matsuzaki H, Iwata N, Xuan M, Kamiuchi S, Hibino Y, Sakamoto T, Okazaki M: Protective Effects of Ferulic Acid against Chronic Cerebral Hypoperfusion-Induced Swallowing Dysfunction in Rats. Int J Mol Sci 2017;18:

24 Nakagawa T, Ohrui T, Sekizawa K, Sasaki H: Sputum substance P in aspiration pneumonia. Lancet 1995;345:1447.

25 Arai T, Yoshimi N, Fujiwara H, Sekizawa K: Serum substance P concentrations and silent aspiration in elderly patients with stroke. Neurology 2003;61:1625-1626.

-26 Suntrup-Krueger S, Bittner S, Recker S, Meuth SG, Warnecke T, Suttrup I, Marian T, Dziewas R: Electrical pharyngeal stimulation increases substance P level in saliva. Neurogastroenterol Motil 2016;28:855-860.

27 Suntrup S, Marian T, Schroder JB, Suttrup I, Muhle P, Oelenberg S, Hamacher C, Minnerup J, Warnecke T, Dziewas R: Electrical pharyngeal stimulation for dysphagia treatment in tracheotomized stroke patients: a randomized controlled trial. Intensive Care Med 2015;41:1629-1637.

28 Warnecke T, Suntrup S, Teismann IK, Hamacher C, Oelenberg S, Dziewas R: Standardized endoscopic swallowing evaluation for tracheostomy decannulation in critically ill neurologic patients. Crit Care Med 2013;41:1728-1732.

29 Dziewas R, Mistry S, Hamdy S, Minnerup J, Van Der Tweel I, Schabitz W, Bath PM, Investigators P-T: Design and implementation of Pharyngeal electrical Stimulation for early de-cannulation in TRACheotomized (PHAST-TRAC) stroke patients with neurogenic dysphagia: a prospective randomized single-blinded interventional study. Int J Stroke 2016;10.1177/1747493016676618

-30 Bath PM, Scutt P, Love J, Clave P, Cohen D, Dziewas R, Iversen HK, Ledl C, Ragab S, Soda H, Warusevitane A, Woisard V, Hamdy S, Swallowing Treatment Using Pharyngeal Electrical Stimulation Trial I: Pharyngeal Electrical Stimulation for Treatment of Dysphagia in Subacute Stroke: A Randomized Controlled Trial. Stroke 2016;47:1562-1570.

-31 Sporns PB, Muhle P, Hanning U, Suntrup-Krueger S, Schwindt W, Eversmann J, Warnecke T, Wirth R, Zimmer S, Dziewas R: Atrophy of Swallowing Muscles Is Associated With Severity of Dysphagia and Age in Patients With Acute Stroke. J Am Med Dir Assoc 2017;10.1016/j.jamda.2017.02.002

-32 Adams HP, Jr., Bendixen BH, Kappelle LJ, Biller J, Love BB, Gordon DL, Marsh EE, 3rd: Classification of subtype of acute ischemic stroke. Definitions for use in a multicenter clinical trial. TOAST. Trial of Org 10172 in Acute Stroke Treatment. Stroke 1993;24:35-41.

-33 Nakato R, Manabe N, Shimizu S, Hanayama K, Shiotani A, Hata J, Haruma K: Effects of Capsaicin on Older Patients with Oropharyngeal Dysphagia: A Double-Blind, Placebo-Controlled, Crossover Study. Digestion 2017;95:210-220.

34 Fraser C, Rothwell J, Power M, Hobson A, Thompson D, Hamdy S: Differential changes in human pharyngoesophageal motor excitability induced by swallowing, pharyngeal stimulation, and anesthesia. Am J Physiol Gastrointest Liver Physiol 2003;285:G137-144.

-35 Hamamoto T, Takumida M, Hirakawa K, Tatsukawa T, Ishibashi T: Localization of transient receptor potential vanilloid (TRPV) in the human larynx. Acta Otolaryngol 2009;129:560-568.

-36 Alvarez-Berdugo D, Rofes L, Casamitjana JF, Padron A, Quer M, Clave P: Oropharyngeal and laryngeal sensory innervation in the pathophysiology of swallowing disorders and sensory stimulation treatments. Ann N Y Acad Sci 2016;1380:104-120.

-37 Jayasekeran V, Singh S, Tyrrell P, Michou E, Jefferson S, Mistry S, Gamble E, Rothwell J, Thompson D, Hamdy S: Adjunctive functional pharyngeal electrical stimulation reverses swallowing disability after brain lesions. Gastroenterology 2010;138:1737-1746.

-38 Vasant DH, Michou E, O'Leary N, Vail A, Mistry S, Hamdy S, Greater Manchester Stroke Research N: Pharyngeal Electrical Stimulation in Dysphagia Poststroke: A Prospective, Randomized Single-Blinded Interventional Study. Neurorehabil Neural Repair 2016;30:866-875.

39 PHADER European Registry. PHAryngeal electrical stimulation for treatment of neurogenic Dysphagia: a European Registry (PHADER). Phagenesis Limited; 2014 [updated: October 15 ${ }^{\text {th }} 2014$; cited 2017 September $26^{\text {th }}$; Version 1.0: [Clinical Investigation Plan Number: AHE-02]. Available from: http://www. phagenesis.com/wp-content/uploads/2014/12/AHE02-CIP-SYNOPSIS-PHADER.pdf. 


\section{SiGNAis}

Neurosignals 2017;25:74-87

\begin{tabular}{l|l}
\hline DOI: 10.1159/000482002 & $\begin{array}{l}\text { C } 2017 \text { The Author(s). Published by S. Karger AG, Basel } \\
\text { www.karger.com/nsg }\end{array}$ \\
\begin{tabular}{l} 
Published onIIne: October 17, 2017 \\
\hline
\end{tabular}
\end{tabular}

Muhle et al.: SP Concentration Following PES in Dysphagic Stroke Patients

40 ISRCTN registry. A European registry to observe the use of the Phagenyx devices for the treatment of patients with swallowing difficulties. 2014 [cited 2017 September 26 $6^{\text {th }}$; Available from: http://www. isrctn.com/ISRCTN87110165. DOI 10.1186/ISRCTN87110165.

41 Scutt P, Lee HS, Hamdy S, Bath PM: Pharyngeal Electrical Stimulation for Treatment of Poststroke Dysphagia: Individual Patient Data Meta-Analysis of Randomised Controlled Trials. Stroke Res Treat 2015;2015:429053.

42 Restivo DA, Casabona A, Centonze D, Marchese-Ragona R, Maimone D, Pavone A: Pharyngeal electrical stimulation for dysphagia associated with multiple sclerosis: a pilot study. Brain Stimul 2013;6:418-423.

-43 Warnecke T, Ritter MA, Kroger B, Oelenberg S, Teismann I, Heuschmann PU, Ringelstein EB, Nabavi DG, Dziewas R: Fiberoptic endoscopic Dysphagia severity scale predicts outcome after acute stroke. Cerebrovasc Dis 2009;28:283-289.

44 Jeyaseelan RD, Vargo MM, Chae J: National Institutes of Health Stroke Scale (NIHSS) as An Early Predictor of Poststroke Dysphagia. PM R 2015;7:593-598.

45 Okubo PC, Fabio SR, Domenis DR, Takayanagui OM: Using the National Institute of Health Stroke Scale to predict dysphagia in acute ischemic stroke. Cerebrovasc Dis 2012;33:501-507.

46 Tomaki M, Ichinose M, Miura M, Hirayama Y, Kageyama N, Yamauchi H, Shirato K: Angiotensin converting enzyme (ACE) inhibitor-induced cough and substance P. Thorax 1996;51:199-201.

47 Arai T, Yasuda Y, Takaya T, Toshima S, Kashiki Y, Yoshimi N, Fujiwara H: ACE inhibitors and symptomless dysphagia. Lancet 1998;352:115-116.

-48 Liu CL, Shau WY, Wu CS, Lai MS: Angiotensin-converting enzyme inhibitor/angiotensin II receptor blockers and pneumonia risk among stroke patients. J Hypertens 2012;30:2223-2229.

49 Sekizawa K, Matsui T, Nakagawa T, Nakayama K, Sasaki H: ACE inhibitors and pneumonia. Lancet 1998;352:1069.

50 Caldeira D, Alarcao J, Vaz-Carneiro A, Costa J: Risk of pneumonia associated with use of angiotensin converting enzyme inhibitors and angiotensin receptor blockers: systematic review and meta-analysis. BMJ 2012;345:e4260.

51 Ohkubo T, Chapman N, Neal B, Woodward M, Omae T, Chalmers J, Perindopril Protection Against Recurrent Stroke Sutdy Collaborative G: Effects of an angiotensin-converting enzyme inhibitor-based regimen on pneumonia risk. Am J Respir Crit Care Med 2004;169:1041-1045.

52 Lee JS, Chui PY, Ma HM, Auyeung TW, Kng C, Law T, Ng LK, Tam KF, Tang WH, Chan BY, Tong MC, Wong KT, Yuen YH, Yuk KL, Kwok T: Does Low Dose Angiotensin Converting Enzyme Inhibitor Prevent Pneumonia in Older People With Neurologic Dysphagia--A Randomized Placebo-Controlled Trial. J Am Med Dir Assoc 2015;16:702-707.

53 Liu CL, Shau WY, Chang CH, Wu CS, Lai MS: Pneumonia risk and use of angiotensin-converting enzyme inhibitors and angiotensin II receptor blockers. J Epidemiol 2013;23:344-350.

54 Dublin S, Walker RL, Jackson ML, Nelson JC, Weiss NS, Jackson LA: Angiotensin-converting enzyme inhibitor use and pneumonia risk in community-dwelling older adults: results from a population-based case-control study. Pharmacoepidemiol Drug Saf 2012;21:1173-1182. 